\title{
Apprentissage intuitif des couleurs à l'aide de la visualisation pictographique simultanée
}

Christelle Morisset-Dammann, Céline Bichon et Gérald Schlemminger

\section{(2) OpenEdition \\ 12 Journals}

Édition électronique

URL : http://journals.openedition.org/esp/3853

DOI : 10.4000/esp.3853

ISSN : 2532-0319

Éditeur

Centre d'Information sur l'Éducation Bilingue et Plurilingue

Édition imprimée

Date de publication : 1 juin 2019

Pagination : 49-57

ISSN : 1127-266X

\section{Référence électronique}

Christelle Morisset-Dammann, Céline Bichon et Gérald Schlemminger, « Apprentissage intuitif des couleurs à l'aide de la visualisation pictographique simultanée », Éducation et sociétés plurilingues [En ligne], 46 | 2019, mis en ligne le 01 février 2021, consulté le 02 mars 2021. URL : http://

journals.openedition.org/esp/3853; DOI : https://doi.org/10.4000/esp.3853

Ce document a été généré automatiquement le 2 mars 2021.

(C) CIEBP 


\title{
Apprentissage intuitif des couleurs à l'aide de la visualisation pictographique simultanée
}

\author{
Christelle Morisset-Dammann, Céline Bichon et Gérald Schlemminger
}

1 L'enseignement de l'accord des adjectifs de couleur au sein du groupe nominal pose à l'apprenant un certain défi car cette règle de grammaire relève d'une certaine complexité relativement abstraite pour des apprenants débutants. Dans cet article, nous proposons une approche plus intuitive, directe et basée sur une métaphore très répandue: les couleurs chaudes et froides.

2 Dans notre démarche, nous nous appuyons sur une approche sensible et directe. Déjà, Heribert Rück (2004) a démontré l'importance du son, du rythme et du mouvement dans l'enseignement des langues étrangères, en prenant en compte les impressions sensorielles élémentaires. Dans un sens plus large, Célestin Freinet (1950), entre autres, parlait d'une psychologie sensible. Une telle approche sensible et directe permet d'aborder des notions abstraites comme la réflexion sur la langue (la grammaire). Gérald Schlemminger (1993) a approfondi cette notion pour l'enseignement des langues.

3 Nous allons présenter dans un premier temps comment les couleurs sont introduites au tout-débutants du primaire au moyen de la méthode Carte-Mot-Dessin (MorissetDammann 2013) et de la classification des notions ressenties de couleurs comme étant froides ou chaudes. Par la suite, nous démontrerons la facilité de transmettre la structure linguistique de l'accord des adjectifs de couleurs dans le groupe nominal, au moyen d'un tableau et d'une métaphore propre à la méthode Carte-Mot-Dessin.

\section{Niveau 1, débutants}

4 L'année scolaire commence et nous sommes face à des enfants débutants (6 à 9 ans), curieux d'apprendre une nouvelle langue. L'apprentissage facile du français en primaire devient un défi passionnant à relever. Nous nous attachons dans cet article à 
montrer de façon très simple l'utilisation des adjectifs de couleurs dans le groupe nominal.

Pour mieux faire comprendre le programme scolaire en français du primaire, nous utilisons l'image d'un grand TGV composé d'une locomotive comprenant deux thèmes de base, les chiffres et les couleurs, et de douze wagons accrochés les uns aux autres, correspondant aux douze thèmes recommandés généralement dans les instructions officielles: l'année - la famille - le corps - les vêtements - les animaux - les fruits et légumes - la maison - les meubles - les moyens de transport - la ville - le restaurant l'heure.

6 L'introduction des deux thèmes de base (les chiffres et les couleurs) de la locomotive ne nécessite pas forcément beaucoup d'heures de cours, puisqu'ils vont être repris systématiquement dans tous les thèmes suivants.

7 Sachant que les enfants apprécient les bandes dessinées, les albums, les petites histoires, il est conseillé d'y avoir recours pour illustrer de façon simple les régularités et le fonctionnement d'une langue. De plus, l'introduction du fonctionnement d'une langue avec humour est un moyen supplémentaire de permettre aux enfants, au travers de leurs émotions positives, de mieux retenir des faits de langue.

\section{Introduction du vocabulaire des couleurs}

Nous présenterons dans cette partie la méthode de la visualisation pictographique simultanée. Nous disposons de cartes-dessins et de cartes-mots, de petite et de grande taille (format A6 et A7, voir figure 1) et de deux planches A3 regroupant les images et les mots des couleurs, qui vont nous servir dès le premier cours (pour toute la méthode, voir Morisset-Dammann 2015). Tous assis en cercle autour de ces deux planches placées au sol, nous faisons découvrir aux enfants une couleur après l'autre, au moyen des cartes-dessins et des cartes-mots.
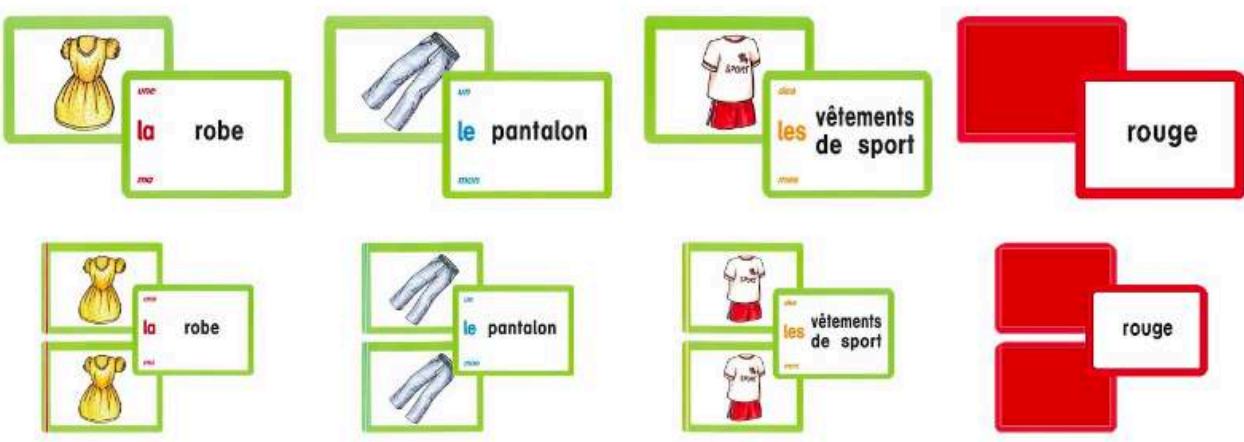

Figure 1: cartes-dessins et cartes-mots

\section{1 ère étape}

Nous tenons dans la main trois petites cartes: deux cartes-dessins suivies d'une cartemot correspondant à une seule couleur, par exemple le rouge. Nous posons la question: "Qui connaît cette couleur?». Les enfants répondent souvent en chœur en allemand «rot!» puisqu'ils ne connaissent pas encore le mot français. Mais ce n'est pas grave, le 
principal est leur attention au jeu. Le premier à avoir répondu a par conséquent le droit de déposer la première carte-dessin du rouge sur la grande planche-dessins.

\section{$2^{\text {ème }}$ étape}

10 Nous utilisons la deuxième carte-dessin pour annoncer le nom de la couleur en français: «Es klingt fast gleich, Kinder: rouge!» et nous montrons simultanément la cartemot du mot "rouge".

Dans un premier temps, les enfants photographient des yeux le mot écrit (visualisation de l'orthographe du mot) en l'associant avec le mot parlé (enregistrement de la chaîne phonétique). Dans un deuxième temps, ils visualisent la représentation mentale du mot de la couleur, en l'associant au mot écrit et au mot parlé. Nous nous assurons que le premier enregistrement du mot "rouge" se fait sur trois niveaux simultanément: la vue, l'ouie et le parler.

\section{$3^{\text {ème }}$ étape}

Guidés par la carte-mot, nous recherchons tous ensemble le mot "rouge" correspondant à la carte sur la planche-mots. Il n'est pas question d'évoquer ici ni l'orthographe ni les syllabes présentes dans le mot. Ce n'est qu'une reconnaissance visuelle globale du mot. Dès que le mot "rouge" est retrouvé sur la planche, un élève est désigné pour y placer cette carte-mot.

\section{$4^{\text {ème }}$ étape}

Pour ancrer davantage les associations évoquées à la deuxième étape, nous introduisons maintenant les grandes cartes. Un petit groupe d'élèves cherche dans le tas de grandes cartes les deux correspondant à la couleur rouge, carte-dessin et cartemot, pour les poser sur le tableau noir visible par tous. Puis, nous prononçons de nouveau le mot 'rouge' et toute la classe le répète en cœur.

De la même manière, nous introduisons chaque couleur successivement, ce qui dure une quinzaine de minutes environ.

\section{Couleurs chaudes et froides}

15 Nous sensibilisons les élèves désormais aux notions de couleurs chaudes et froides, afin d'obtenir, plus tard dans l'apprentissage "l'effet de déjà-vu". Ici, il s'agit seulement de préparer les élèves au futur tableau de classification des couleurs (voir figure 2).

Nous associons chaque couleur à un élément ou à une matière différente pour expliquer qu'elle est plutôt froide ou plutôt chaude. Nous faisons alors appel à leur capacité sensorielle du toucher, ou à leur ressenti physique pour illustrer la notion du froid ou $\mathrm{du}$ chaud (cependant ceci ne correspond pas au cercle chromatique des couleurs chaudes ou froides en art graphique).

17 Nous introduisons le sujet des couleurs au moyen d'une métaphore. Dans notre classification, les couleurs froides sont le blanc - comme la neige, le noir - comme la nuit, le bleu - comme l'eau, le vert - comme l'herbe, le gris - comme les nuages et le 
violet - comme un ciel orageux ou un cristal. Les couleurs chaudes sont le rouge comme le sang, le jaune - comme le soleil, le rose comme la langue.

Pour renforcer la sensibilisation aux deux catégories de couleurs principales (chaudes et froides), nous employons les mimes et la grande planche de dessins des couleurs. Nous désignons du doigt une couleur et demandons au moyen de mimes aux élèves: " chaud? ", avec le dos de la main sur le front en soufflant très fort et en disant «quelle chaleur!». Pour le froid, nous nous tenons le corps en nous frictionnant les bras et en sautillant d'un pied à l'autre. Les élèves se rappelant du ressenti de la couleur, miment la réponse correctement. Nous pouvons également utiliser les cartes-dessins (ou cartesmots) pour inciter ces mimes. Il suffit de montrer une carte-mot ou une carte-dessin à toute la classe et d'attendre que les élèves miment correctement la catégorie de la couleur représentée par celle-ci.

Suite à quoi, nous pouvons demander aux élèves « Qu'est-ce que c'est? » en désignant une seule couleur sur la planche imagée. Ils doivent tous annoncer le mot avant de répéter les gestes et mimes en rapport avec les couleurs " chaudes et froides », sans se tromper.

\section{Niveau 2}

$\mathrm{Au}$ cours de la deuxième année, nous reprenons la notion de couleurs chaudes et froides en y ajoutant les exceptions. Rappelons les règles que nous avons tous apprises au lycée concernant l'accord des couleurs:

1. Les adjectifs de couleurs s'accordent en genre et nombre avec le nom qu'ils qualifient.

2. La règle stipule que tous les noms de fleurs (le lilas...), de pierres précieuses (turquoise...) et de fruits (le kaki, le marron, l'orange) utilisés pour qualifier une couleur restent invariables, par exemple une jupe marron.

3. Il y a des exceptions à cette règle, par exemple la couleur rose, qui est un nom de fleur, qui devrait être invariable, s'accorde néanmoins au pluriel avec le nom qu'il qualifie, exemple les « chaussettes roses ».

21 Cette règle est évidemment bien trop complexe pour l'aborder à l'école primaire, car les élèves sont encore incapables de reconnaitre le vocabulaire des fruits, des fleurs ou des pierres précieuses en français. Il ne faut pas oublier que nous ne sommes qu'au début de la deuxième année de l'apprentissage du français.

Il est vrai que les instructions, en général, ne demandent pas de maîtriser ce fait de langue en primaire. Cependant, si les élèves le demandent, ou bien, si nous voulons relever personnellement le défi de faire comprendre correctement, dès le début, cette structure linguistique de l'accord des adjectifs de couleurs dans le groupe nominal, il y a une possibilité très simple et très humoristique de le faire. 


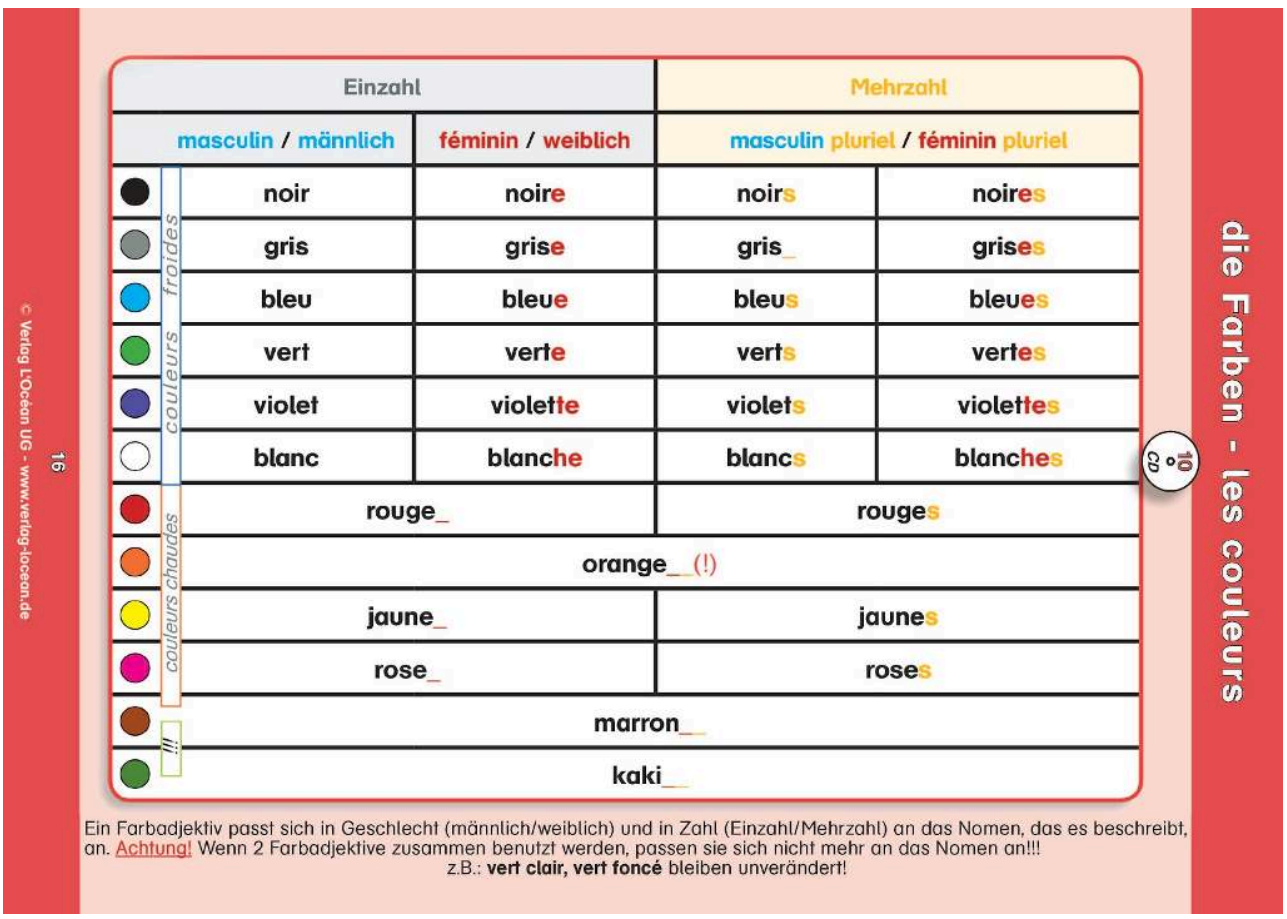

Figure 2: tableau des couleurs

L'adjectif de couleur s'accorde, en général, en genre (féminin/masculin) et en nombre (singulier/pluriel) au nom qu'il qualifie. Attention: lorsqu'il deux adjectifs de couleur sont utilisés ensemble, ils ne s'accordent pas au nom. Exemple: 'vert clair', 'vert foncé' restent invariables.

En observant le tableau de la méthode Carte-Mot-Dessin illustrant la métaphore des couleurs chaudes et froides, nous constatons qu'une première catégorie d'adjectifs de couleur change de terminaison au féminin, au masculin pluriel et au féminin pluriel. Il se trouve que ce sont justement les couleurs que nous avons appelées "froides". Nous les associons (arbitrairement) au fait que l'accord avec le nom s'effectue toujours. Pour expliquer cette association et soutenir la mnémotechnique, nous utilisons la métaphore suivante: lorsque vous avez froid, vous ne restez pas sur place, vous bougez dans tous les sens pour vous réchauffer! Pour ces "couleurs froides", c'est pareil: les terminaisons changent toujours, au féminin, au masculin pluriel et au féminin pluriel.

La deuxième catégorie d'adjectifs de couleur change de terminaison seulement en passant au pluriel; ce sont les couleurs que nous avons appelées "chaudes". Nous avons recours à la même métaphore: lorsqu'il fait chaud, on ne bouge qu'un minimum. Pour ces "couleurs chaudes", c'est pareil: les terminaisons ne changent qu'au pluriel en prenant un 's'.

Une dernière catégorie d'adjectifs de couleur reste invariable. Comment l'appeler?

Pour amener l'explication de l'accord d'adjectifs de couleur chez des élèves débutants en primaire, nous recourons à la langue allemande pour être compris de tous. En ce qui concerne les couleurs froides, nous nous adressons aux élèves avec cette question:

„Was macht ihr, wenn es euch sehr kalt ist? Ihr bleibt an Ort und Stelle, ohne euch zu bewegen? Nein! Ihr bewegt euch in alle Richtungen, um euch aufzuwärmen! (Pantomim von jemanden, der von einem Fuß auf den anderen springt, indem er seine Arme reibt). Für Farben ist es das Gleiche! Alles ist gut, um sich zu bewegen: der Übergang von weiblich $\mathrm{zu}$ männlich, der von Singular zu Plural ... zum Aufwärmen! Die Farben setzen eine Mütze $(=$ e) vom Männlichen zum Weiblichen, 
einen Schal (= s) vom Singular zum Plural und Handschuhe (= es), während sie zum weiblichen Plural übergehen“

"Que faites-vous, quand vous avez très froid? Vous restez sur place sans bouger? Non! Vous bougez dans tous les sens pour vous réchauffer!" (en mimant quelqu'un qui sautille d'un pied sur l'autre en se frottant les bras). Pour les couleurs, c'est la même chose! Tout est bon pour bouger: le passage du féminin au masculin, du singulier au pluriel... pour se réchauffer! Les couleurs mettent un bonnet (le 'e') en passant du masculin au féminin, une écharpe (le 's') en passant du singulier au pluriel et des gants ('es') en passant au féminin pluriel. suivante : schreibt und ein Gesicht macht" invariables. classe éclate de rire en cœur! froides, chaudes et « cacas ». retrouver le bon accord.

Pour introduire les couleurs chaudes, nous racontons de façon interactive l'histoire

„Was macht ihr, wenn es euch heiß ist? Lauft ihr in alle Richtungen, wenn es draußen $38^{\circ} \mathrm{C}$ ist/gibt? Nein! Ihr bewegt euch bestimmt nicht in alle Richtungen, um euch aufzuwärmen! (Pantomim von jemanden, der sich langsam bewegt und seine Hand auf die Stirn legt ). Für die Farben ist es ähnlich! Sie bewegen sich nur für das absolute Minimum! Das ist der Übergang vom Singular zum Plural ... und das ist genug für sie! Die Farben, nachdem sie vom Singular zum Plural gegangen sind, müssen sich sofort mit einem Wasserschlauch abkühlen $(=\mathrm{s})^{\text {“ }}$

Que faites-vous, quand vous avez très chaud? Vous courez dans tous les sens? Non! Vous faites le strict minimum! (en mimant quelqu'un qui se passe le dos de la main sur le front en signe de forte chaleur). Pour les couleurs, c'est la même chose! Cela leur donne déjà très chaud de passer du singulier au pluriel et cela leur suffit largement! Les couleurs prennent alors un tuyau d'arrosage $(=s)$ pour se refroidir!

Pour introduire les couleurs invariables dites "caca" nous avons délibérément choisi la métaphore enfantine suivante, qui amène inévitablement le fou rire dans le cours. Nous nous adressons aux élèves avec l'histoire suivante :

„Ich habe einen Hund und meine Eltern haben Gänse. Braun ist die Farbe des Hundes "Kaka" und Khaki ist die Farbe des Gänse "Kaka"! ... Wer kehrt gerne mit seinem Stift in 'Kaka' zurück, um etwas zu ändern oder hinzuzufügen? So bleiben diese Farben unveränderlich! ... Pantomime von jemandem, der in die Leere

J'ai un chien à la maison et mes parents ont des oies. Marron est la couleur du caca de chien et kaki est la couleur du caca d'oie. Dites-moi, qui retourne volontiers dans le caca avec son crayon pour y changer quelque chose ? Ces couleurs restent donc

Maintenant arrive la question automatique et rhétorique des enfants: „Und von wem kommt das orangene Kaka?" (et orange ? C'est le caca de qui ? [car invariable aussi...]) La

Mais le plus important de cette métaphore est que le fait de langue soit visualisé et compris. Les élèves qui doivent utiliser par la suite une couleur dans un groupe nominal choisissent celle-ci en se remémorant cette histoire drôle sur les couleurs

D’après notre expérience d'enseignante, les élèves optent généralement dans un premier temps, pour une couleur invariable parce que c'est drôle et facile... puis dans un deuxième temps, les couleurs chaudes, parce qu'elles ne prennent qu'un 's' au pluriel . Les plus ambitieux se risqueront même à utiliser une couleur froide malgré les changements orthographiques, sachant qu'ils sont autorisés à consulter le tableau pour 


\section{BIBLIOGRAPHIE}

FREINET C. 1950. Essai de psychologie sensible appliquée à l'éducation. Cannes: CEL.

MENTZ O. 2003. Baustelle Französisch. Erwartungen an die Grundschule und Impulse durch frühes Fremdsprachenlernen, Französisch heute, 34, 2003/ 2: 132-139.

MORISSET-DAMMANN C. 2018. La méthode 'Carte-mot-dessin' (CMD), Freiburg i. Br.: Verlag L’Océan (première édition: 2013).

RÜcK H. 2004. Fremdsprachen in der Grundschule: Französisch und Englisch, Landau: KnechtVerlag.

SCHLEMMINGER G.1993. Le point sur les méthodes naturelles, la correspondance et l'enseignement des langues vivantes, Tracer, revue d'innovation et de recherches en enseignement des langues, $n^{\circ} 2$ (juin): 3-7.

\section{RÉSUMÉS}

L'obiettivo dell'articolo è di spiegare l'apprendimento intuitivo dell'accordo degli aggettivi tramite la visualizzazione grafica simultanea e l'uso di metafore e basandoci su un approcchio sensibile e diretto. Un simile approccio permette di affrontare nozioni astratte come la riflessione sulla lingua (grammatica). Presenteremo un un primo tempo come i colori vengano introdotti ai principianti della scuola primaria. In seguito mostreremo come sia facile trasmettere la struttura linguistica dell'accordo degli aggettivi designanti i colori all'interno del gruppo nominale grazie ad una tabella e ad una metafora propria del metodo Carta-ParolaDisegno.

The aim of this article is to explain how the agreement of adjectives can be learned intuitively thanks to the simultaneous pictographic method and to metaphors, by using a sensitive and direct approach. Such an approach permits broaching abstract notions such as thinking about language (grammar). We will first present the ways colors are introduced in a complete beginners' class. We will next demonstrate how simple it is to transmit the linguistic structure of the agreement of color adjectives in the noun phrase, thanks to a metaphor taken from the MapWord-Drawing method. 
INDEX

Parole chiave : apprendimento intuitivo, grammatica, visualizzazione grafica simultanea, approccio sensibile, aggettivi di colori, metodo

Keywords : intuitive learning, grammar, simultaneous pictographic visualizing, sensitive approach, color adjectives, method

\section{AUTEURS}

\section{CHRISTELLE MORISSET-DAMMANN}

Pädagogishe Hochschule, Karlsruhe (Allemagne)

\section{CÉLINE BICHON}

Pädagogishe Hochschule, Karlsruhe (Allemagne)

\section{GÉRALD SCHLEMMINGER}

Pädagogishe Hochschule, Karlsruhe (Allemagne) 